\title{
ALTA HOSPITALAR DA CRIANÇA: IMPLICAÇÕES PARA A ENFERMAGEM
}

THE CHILD'S DISCHARGE: IMPLICATIONS FOR NURSING

Lelia Maria Madeira $^{1}$

MADEIRA, L. M. Alta Hospitalar da Criança: Implicações para a Enfermagem. Rev. Boas. Cresc. Des. Hum., São Paulo, IV(2), 1994.

Resumo: Focalizando a alta da criança, a autora descreve os malefícios da hospitalização, a importancia da alta planejada, bem como do seguimento pós-alta da criança, no domicílio; apresenta as implicações para a enfermagem e discute o- papel do enfermeiro neste processo.

Palavras-Chave: Criança hospitalizada, alta hospitalar, enfermagem pediátrica, assistência à criança, seguimento pós-alta.

Summary: Focusing on lhe child's discharge, this paper describes the harrnful effects of hospitalization, lhe importance of lhe planned discharge, as well as lhe follow-up after discharge by doing home visiting; it presente lhe implications for nursing and discasses lhe role of lhe curse in that process.

Key-words: Hospitalized child, hospital discharge, pediatric nursing, child assistance, follow-up.

1 Prof. adjunto da Disciplina de Enfermagem Pediátrica da Escola de Enfermagem da Universidade Federal de Minas Gerais; Mestre em Emfermagem Pediátrica, Doutoranda do Programa de Doutorado. Convênio EEUSP - EEUFMG. End.: Rua Aurélio Dolabela 14O, Floramar, Belo Horizonte - MG, CEP 31760-470 Fone/Fax: (031) 433-1710. 


\section{INTRODUÇÃO}

Nas últimas décadas, têm ocorrido grandes avanços na assistência à saúde da criança. Novas tecnologias têm surgido, em sua maioria, voltadas para a assistência hospitalar, sendo utilizadas para a recuperação da saúde.

Presenciamos no cotidiano de saúde infantil um grande número de crianças que são hospitalizadas por diferentes motivos, sendo a causa desta, muitas vezes, evitável. Estas crianças quando hospitalizadas, ficam expostas à uma série de riscos, tanto do ponto de vista físico, quanto psicológico. Isto sem considerar que, ao retornarem aos domicílios, a maioria será submetida às condições de vida as mais hostis, o que contribui para sua "re-hospitalização".

No Brasil, segundo ZILIO11O (1992), os brasileiros enfrentam sérios problemas: $70 \%$ da população encontra-se em situação de pobreza e/ ou miséria absoluta, enquanto $50 \%$ do produto interno bruto está nas mãos de apenas $10 \%$ da população. Como se verifica, as désigualdades económicas aliadas a outras adversidades apresentam-se como um grande desafio.

O sistema de saúde, por sua vez, encontra-se desestruturado, incapaz de atender com eficiência a grande parcela da população que dele depende. Dos recursos existentes, o hospital desempenha um importante papel, considerando-se as condições de saúde da população e o enfoque curativo que ainda prevalece no sistema de saúde brasileiro. Em se tratando da assistência à criança, o número de leitos é, frequentemente, insuficiente para atender à demanda; são comuns as internações e reinternações evitáveis; além da prolongada permanência hospitalar, muitas vezes, provocada por iatrogenias.

BERNBAUN et al (1989), descrevendo a problemática da prematuridade nos Estados Unidos, diz que a sobrevivência de crianças prematuras e de baixo peso tem aumentado muito em decorrência dos avanços tecnológicos na assistência neonatal. No entanto, pouco progresso foi feito no sentido de reduzir a incidência de prematuridade e baixo peso ao nascer. Destas crianças, alta porcentagem compõem o grupo de crianças com problemas médicos, neurológicos e de desenvolvimento, designadas por autores, como HOSTLER (1991), como crianças com necessidades de saúde especiais.

LIPSI et al (1991) também se referem às crianças que passam por hospitalizações prolongadas ou "re-hospitalizações" como aquelas que estão sob risco de atrasos desenvolvimentais e, por isso, possuem necessidades especiais.

Para HOSTLER (1991) crianças com necessidades especiais são aquelas que possuem doenças crónicas, deficiências físicas e distúrbios desenvolvimentais, estimadas em 10 a $15 \%$ da população americana com menos de 21 anos de idade. O autor afirma que a sobrevivência destas crianças tem aumentado nos últimos anos, sendo que cerca de $90 \%$ delas estão alcançando a vida adulta. Por outro lado, suas necessidades de saúde são tão complexas que o próprio sistema se sente inapropriado para atendê-las.

Preocupados com esta situação, profissionais responsáveis pela assistência à criança propõem novas abordagens, numa tentativa de minimizarem as conseqüências decorrentes da hospitalização da mesma. Neste sentido, vários autores destacam a importância e a necessidade da alta planejada da criança, bem como de seu acompanhamento pós-alta, no domicílio.

Dentro da realidade brasileira, são raras as experiências relatadas sobre a alta hospitalar da criança e, muito menos, sobre o destino da mesma, após a alta.

Considerando-se as adversidades das condições de vida da maior parte da população brasileira e a situação de saúde infantil determinada pelas múltiplas variaveis contextuais, questionamos:

- Como tem ocorrido a alta de crianças nas instituições hospitalares?

- Como é feito o acompanhamento destas crianças após a alta?

Acreditando ser o enfermeiro, devido as atividades desempenhadas junto à criança, o elemento central da equipe responsável pela assistência hospitalar e pelo acompanhamento pós-alta, indagamos:

- Como tem sido a atuação do enfermeiro na alta hospitalar e no seguimento pós-alta da criança?

Numa tentativa de responder a estas e a outras questões, nosso trabalho propõe-se:

- descrever a problemática que envolve a alta hospitalar da criança;

- refletir sobre a atuação do enfermeiro na alta e no seguimento pós-alta da criança.

\section{A CRIANÇA NO HOSPITAL E SUAALTA}

Seria difícil discorremos sobre questões relativas à alta hospitalar da criança sem, anteriormente, nos reportarmos às possíveis conseqüências da hospitalização para a mesma.

Diversos autores vêm estudando os prejuízos da hospitalização para a criança e, nos últimos tempos, tem havido um empenho dos profissionais da saúde em favor da presença do acompanhaste (mãe, pai, outro) na uni 
dade pediátrica, numa tentativa de minimizar os efeitos maléficos da hospitalização para a criança, MADEIRA (1989) diz que a hospitalização, dependendo da forma como é conduzida, pode trazer graves problemas para a vida da criança e, ao defender o sistema de internação conjunta, expõe as vantagens da mesma para a criança, para a mãe e para a instituição hospitalar.

Atualmente, tem havido uma forte tendência dos profissionais ligados à assistência à criança quanto a proposta de cuidado centrado na família. Para LADEBAUCHE (1992), qualquer hospitalização é estressante para a criança e para sua família. A perda de controle, o medo de traumatismo corporal, a dor, o stress da separação da família tanto quanto as incertezas quanto ao tratamento, contribuem para a ansiedade da criança e da família. Neste sentido, o foco da assistência à criança tem, gradualmente, mudado do cuidado à criança para o cuidado centrado na família. Do mesmo modo, CALLERY \& SMITH (1991), ao discutirem o papel dos pais e do enfermeiro na assistência à criança hospitalizada, dizem que existe hoje uma expectativa de que os pais se tornem profundamente envolvidos no cuidado do filho hospitalizado.

Para HOSTLER (1991), “o cuidado centrado na família é uma nova filosofia de cuidado que coloca a família, ao invés do hospital e da equipe médica, no centro do sistema de prestação de assistência”. A autora af rma que a implementação desta forma de assistência requer mudança de atitude da família e dos profissionais da saúde tanto quanto exige reformulação da política e prática de saúde existentes. Dentro desta visão mais ampia da assistência hospitalar à criança procuraremos focalizar a alta.

Para MARRA et ai (1989), entende-se por alta a condição que permite a saida do paciente do hospital. É um procedimento que engloba todas as maneiras pela qual o paciente pode deixar o hospital.

Sendo assim, qual o momento adequado para a alta?

Profissionais da saúde, considerando as múltiplas conseqüências da hospitalização sobre a criança, têm advogado a favor da alta precoce. Outra justificativa aplicável, especialmente às instituições hospitalares de pa ses em desenvolvimento, é a que diz respeito à superlotação dos hospitais. Neste caso, a alta precoce favoreceria o maior aproveitamento do leito hospitalar (GONZALEZ \& PEROZO, 1987; MARRA et al, 1989).

Entretanto, para a efetividade do tratamento os autores também afirmam que, ao receber a alta hospitalar, todo paciente deve receber orientação quanto à continuidade de seu tratamento e que, quanto mais precoce for a alta mais bem elaborada deve ser a orientação.
BAKER et ai (1989); LADDEN (1990) e BERNBAUM et ai (1989) ao discorrerem sobre a problemática da criança prematura, de baixo peso, portadora de deficiência que a inclui no grupo de crianças com necessidades especiais, reforçam a importância do envolvimento da família e destacam o impacto que a alta destas crianças provoca nas mesmas.

LADDEN (1990) afirma que a alta, para muitas crianças, não significa que sua recuperação está completa e sim que será apenas mudado o local de recuperação do hospital para o domicilio. Isto, muitas vezes, requer uma quantidade enorme de energia física e emocional dos pais que podem se sentir exaustos e isolados ao cuidarem dessa criança 24 horas por dia.

BAKER et ai (1989) dizem que a chegada de uma criança com necessidades especiais altera o estilo de vida da família em muitos aspectos. A necessidade de mais tempo disponível para cuidar da criança implica em que um dos pais abandone o emprego; atividades sociais devem ser diminuídas, além dos outros filhos que, muitas vezes, ficam relegados a segundo plano. Tudo isso, gera uma quantidade de stress muito grande, afetando todos os membros da família.

BERNBAUN et ai (1989) focalizam a criança que sai de alta com cuidados especiais e, às vezes, dependendo de algum tipo de tecnologia, como por exemplo: oxigenoterapia, traqueostomia, dieta por gavage, dentre outras. Os autores dizem que, devido às suas necessidades de saúde, a criança torna-se o centro da família, e as atividades do dia-a-dia são dificultadas. Na maioria das famílias, o cuidado desta criança pode provocar sérios problemas financeiros que, associados aos outros fatores estressantes geram discórdia conjugal, ciúme nos outros filhos, dentre outros. Descrevem a experiência da fantasia no período pós-alta da criança em três estágios:

- o primeiro é de euforia e ocorre nas primeiras seis semanas, quando os pais se sentem superfelizes por terem o filho em casa; finalmente se sentem pais;

- o segundo é representado pela falta de esperança e ocorre, em geral, entre seis semanas e seis meses após a alta; predomina a exaustão e os pais começam a conscientizar-se de que a criança é mais doente do que eles imaginavam;

- o estágio final é de aceitação; os pais começam a integrar a assistência à criança e a complexidade do tratamento em suas vidas. Aceitando a criança com suas necessidades especificas a família é capaz de reorganizar a sua rotina.

Face à complexidade e importância da hospitalização e alta da criança era de se esperar um grande número de publicações sobre o tema. Entretanto, na literatura nacional, encontramos apenas 4 trabalhos publicados nos 
últimos 10 anos: o de MARRA et ai (1989) que defende a alta hospitalar; o de PEDUZI \& VIETTA(1986) sobre o seguimento de enfermagem pós-alta de pacientes psiquiátricos e os demais sobre aspectos da alta da criança. Destes, o de SEGRE et al (1986) fala sobre os efeitos das modificações de critérios para a alta de recémnascidos de baixo peso e o de ROCHA et ai (1981) sobre o tempo de permanência e critérios de alta para desnutridos, ambos sugerindo autoria de profissionais médicos.

No referencial internacional predominam os autores americanos focalizando a alta de crianças com patologias e deficiências diversas, sendo destacados: HULSEMAM \& LEE (1992); MULLEN \& BUCHANAN (1990); WEN-NBERG \& AHLFORS (1988); BERNBAUN et ai (1989); KONGELBECK (1990); BAKER et ai (1989); AHMANN \& LIERMAN (1992) e LADDEN (1990). Destes, a maioria estuda a alta hospitalar da criança recém-nascida com necessidades especiais; são unanimes ao exporem a problemática destas crianças, o impacto das mesmas sobre a família e a necessidade de se elaborar um plano de alta para cada uma.

\section{O PLANO DE ALTA}

O plano de alta é o instrumento sugerido pelos autores para garantir a continuidade da assistência à criança após sua hospitalização. Os aspectos apresentados no quadro em anexo e discutidos a seguir serão fundamentados nas propostas de BAKER et ai (1989); LADDEN (1990); AHMANN \& LIERMAN (1992) e KONGELBECK (1990), por serem os que apresentam estudo mais aprofundado sobre o tema.

Como mostra o quadro, encontramos muitas semelhanças entre as propostas dos autores, apesar de apresentarem diferentes objetivos devido às diferentes populações enfocadas. Dois aspectos chamam-nos a atenção: todos reforçam a importância do envolvimento da fam 1ia/pais em todas as etapas do plano e, principalmente, nas tomadas de decisão o aspecto mais destacado e valorizado é o educativo.

BAKER et ai (1989) falam sobre o plano de alta, porém, ao discutirem suas etapas caracterizam-no como "plano de ensino para a alta”. E afirmam que esse ensino é parte integrante do processo educativo incluindo a orientação à família acerca do que necessita saber e compreender sobre o estado de saúde e necessidades do filho.

O sucesso do plano de alta, independente do quadro da criança, vai depender dos profissionais envolvidos e, principalmente, do momento de seu início. Concordamos com os autores que dizem que a alta e, obviamente, o plano de alta deve ter início na admissão da criança. E neste momento que a equipe multiprofissional inicia um relacionamento de confiança com a família, obtendo e fornecendo informações sobre a criança. E, então, no momento da admissão que se inicia a la. etapa do plano de alta que os autores denominam de avaliação das necessidades da criança e da família. Esta avaliação é fundamental para a implementação das etapas subsequentes e, principalmente, para se considerar a possibilidade de alta precoce.

Os autores chamam a atenção para a avaliação das necessidades da criança e da família e dizem que esta não deve ser feita de forma simplista, direcionada apenas para necessidades físicas, individuais, como por exemplo: a habilidade da mãe administrar a dieta por sonda, atraso no desenvolvimento motor, dentre outros. E necessário que se faça uma avaliação mais ampla dessas necessidades, considerando-se aspectos emocionais, financeiros e o ambiente familiar. Todos os esforços deverão ser feitos no sentido de integrar a criança recém-hospitalizada ao contexto de sua família, com o mínimo de stress possível. Esta perspectiva reforça a necessidade da continuidade da assistência à criança após a alta.

\section{SEGUIMENTO PÓS-ALTA}

O contexto familiar e o ambiente em que vive a criança têm uma influência marcante na sua recuperação e sobre seu processo de desenvolvimento.

Em geral, a criança com necessidades especiais, que foi tratada no hospital em unidade intensiva, com equipe de especialistas e todo equipamento sofisticado, sofre um grande impacto ao chegar em casa. Muitas vezes, o ambiente domiciliar é completamente adverso e não possui infraestrutura suficiente para receber esta criança.

SCHRAEDER (1986), falando a respeito do aumento de sobrevivência de recém-nascidos devido aos avanços tecnológicos, afirma que o excesso de cuidado e o interesse no bem-estar da criança, frequentemente, terminam na porta do hospital. Em seu estudo, a autora chama a atenção para a incongruência verificada; diz ser inacreditável que estas crianças tenham tido tantos milhões de dólares e tantas horas de profissionais especializados gastos em seu tratamento e saiam do hospital tendo apenas agendado o acompanhamento de rotina de um recém-nascido normal. Das crianças prematuras de baixo peso que acompanhou durante o $1^{\circ}$ ano de vida, 29\% eram filhas de mães solteiras e desempregadas e $24 \%$ provinham de lares onde a mãe ou o pai estava desempregado. Conclui seu trabalho dizendo que, se o desenvolvimento da criança depende de pais que tenham: posses; energia física e emocional para cuidar da criança; conhecimento e recursos para estimularem o desenvolvi- 
mento da criança e habilidades para proporcionar experiências interessantes para o filho, isto significa que dar alta à criança de uma unidade de terapia intensiva é apenas metade do trabalho.

Concordamos plenamente com a autora quando refletimos sobre as condições de vida e saúde de nossa população.

A necessidade de uma avaliação apurada da família e o seguimento domiciliar pós-alta fazse necessário não só em casos de crianças como as citadas pela autora anteriormente. Além destas, temos um número enorme de crianças que se encontram sob risco, não somente pela patologia ou deficiência que portam mas, também, devido àquelas que podem adquirir através das precárias condições de vida a que são submetidas.

Numa tentativa de amenizar estes riscos vários autores sugerem a visita domiciliar periódica para controle de saúde da criança, para dar continuidade ao seu processo de recuperação e, também, apoiar os pais em suas necessidades.

Para BAKER et al (1989) são necessárias visitas domiciliares e acompanhamento por telefone, feitos após a alta de recém-nascidos prematuros com necessidades especiais, para avaliar o progresso da família e ajudar os pais na adequação da assistência à criança no domicílio. Sugerem que estas visitas sejam efetuadas pela equipe multiprofissional da Unidade de Terapia Intensiva Neonatal.

SCHRAEDER et ai (1990); SEGRE et al (1986); GONZALEZ \& PEROZO (1987) e SIQUEIRA et ai (1992), em seus trabalhos, destacam as vantagens da visita domiciliar à criança:

- possibilita a alta precoce, beneficiando a criança e reduzindo custos hospitalares;

- permite a caracterização do ambiente físico; a anarnnese e observação da interação mãecriança, bem como observação, em situação natural, de marcos do desenvolvimento biopsicossocial;- favorece a identificação do risco da criança;

- apresenta o quadro das condições sociais, emocionais e dos recursos disponíveis para a criança.

Tendo em vista a importância da alta hospitalar, a avaliação das necessidades de saúde da criança e da família, a elaboração e implementação do plano de alta bem como o acompanhamento no nível domiciliar, questionamos:

- Qual seria o papel.do enfermeiro neste processo?

\section{IMPLICAÇÕES PARAA ENFERMAGEM}

A criança, ao ser admitida em uma unidade hospitalar, é assistida por uma equipe multidisciplinar. Dependendo de suas necessidades e dos recursos humanos existentes na instituição ela terá, no mínimo, a assistência de membros da equipe médica e de enfermagem. Por isso, ao repetirmos sobre responsabilidades quanto à alta da criança, seria injusto atribuirmos toda ela ao enfermeiro. No entanto, é indiscutível que ele se encontra em posição estratégica quanto aos demais profissionais da equipe, para assumir a coordenação do plano de alta.

E este o papel que os autores atribuem ao enfermeiro, justificando que é ele quem está mais próximo à criança e à família e que possui uma visão mais ampla das necessidades de saúde da criança.

AHMANN \& LIERMAN (1992), em sua proposta de plano para criança com dependência de tecnologia, atribuem ao enfermeiro a coordenação da equipe de implementação do plano de alta destacando suas responsabilidades:

- acompanhar o desenvolvimento de todo o plano de alta;

- especificar as responsabilidades da família e de outros membros da equipe;

- controlar os serviços prestados para garantir o atendimento das necessidades de saúde, a qualidade do cuidado e evitar duplicação de esforços;

- fornecer informações à família sobre os recursos disponíveis na comunidade; - avaliar e rever o plano assistencial, regularmente;

- facilitar a comunicação entre a família e os provedores de cuidados, entre estes e outros, quando necessário.

Estamos de acordo com os autores quanto às atribuições do enfermeiro e acreditamos ser imprescindível seu envolvimento no processo de alta da criança, com vistas à continuidade de assistência no domicílio. Se não nos preocuparmos com o preparo da família, com o ambiente para o qual a criança irá retornar, com certeza não conseguiremos efetividade nos tratamentos hospitalares, mesmo com a utilização da tecnologia avançada.

No entanto, precisamos compreender toda a problemática da enfermagem enquanto profissão, as condições sócio-econômicas de nossa população e, principalmente, as deficiências de nossa formação profissional.

Para obtermos profissionais engajados na prevenção e recuperação da saúde de nossas crianças, numa perspetiva mais ampla: criança-família, hospital-comunidade-domicílio, serão necessárias algumas mudanças e redirecionamentos. Em primeiro lugar, exige-se mudança de atitude do profissional em relação à saúde da criança, 
ou seja, conscientizar-se da importância da hospitalização e da alta para a vida da criança, possuir uma visão integrada da assistência e sentir-se comprometido com seu cliente, advogando em defesa de sua saúde.

Acreditamos que essas mudanças poderão ser viabilizadas através da valorização do assunto e inclusão do mesmo nos currículos de enfermagem, gerando amplas discussões não só sobre os aspectos técnicos mas, principalmente, sobre as conseqüências das condições mórbidas a que estão expostas nossas crianças; do incentivo e expansão de pesquisas sobre o assunto, provocando a socialização do conhecimento e a busca de alternativas para as questões colocadas.

\section{CONCLUSÃO}

Considerando a realidade de vida e saúde da população infantil brasileira constatamos

\section{BIBLIOGRAFIA}

AHMANN, E.; LIERMAN, C. Promoting normal development in technology -dependent child: an introduction lo lhe issues. Pediatr. Nurs., 18(2):1438, 1992.

BAKER, K. et ai. Discharge teaching for parents of newborns with special needs. Nurs. Clin. North Am., 24(3):655-64, 1989.

BERNBAUN, J. C. et ai. Pretern infant care after hospital discharge. Pediatr. Reu, 10(7):195-206, 1989.

CALLERY, R; SMITH, L. A study of role negotiation between nurses and the parente of hospitalized children. J. Adu Nurs., 16:772-81, 1991.

GONZALEZ, E. J.; PEROZO, M. A. de. Alta precoz en recién nascidos nommales. Boi. Med Postgrad., III(3):31-38, 1987.

HOSTLER, S. L. Family-centered care. Pediatr. Clin. North Am., 38(ó):1545-61, 1991.

HULSEMAN, M. L.; LEE, A. L. The neonatal ICU graduate: Part 1. Comnom problems. Am. Fam. Physician, 45(3):1301-5, 1992.

KONGELBECK, S. R. Discharge planning for the child with infanfile spasms. J. Neurosci. Nurs., 22(4):23844, 1990.

LADDEN, $\mathrm{M}$. The impact of preterm birth on $\wedge$ the family and society. Part 2: transition to home. Pediatr. Nurs., 16(ó):620-26, 1990.

LADEBAUCHE, R Unit-based family - support groups: a reminder. MCN, 17:18-21, 1992.

LIPSI, K. et ai. Developmental rounds: an intervention stratregy for hospitalized infants. Pediatr. Nurs., 17(5):433-7, 1991.

MADEIRA, L. M. Intemação conjunta em pediatria: uma visão geral. Belo Horizonte: Escola de Enfemmagem da UFMG, 1989, 13 p. [mimeo], a importancia da hospitalização e, consequentemente, do processo de alta na vida da criança.

A alta de uma criança, em geral, pode gerar distúrbios familiares com conseqüências sérias para a saúde da mesma. Por isso, demanda a atuação de uma equipe multiprofissional visando, principalmente, a manutenção da saúde com a continuidade do tratamento, a diminuição do risco de "re-hospitalização" e o apoio familiar.

Nesta perspectiva é imprescindível o acompanhamento domiciliar da criança no período pós-alta, quando a mesma ainda permanece sob risco.

Concordamos com a maioria dos autores consultados que atribuem ao enfermeiro um papel central neste processo, porém, acreditamos que sua atuação se efetivará à medida que forem ocorrendo mudanças na atitude do profissional e, principalmente, relacionadas à sua formação.

MARRA, C. C. et ai. Orientação planejada de enfermagem na alta hospitalar. Acta. Paul. Enf:, 2(4):123-7, 1989.

MULLEN, C. A.; BUCHANAN, G. R. Early hospital discharge of children with cancer treated for fever and neutropenia: identification and management of lowrisk patient. J. Clin. Oncol., 8(12):1998-2004, 1990.

PEDUZI, M.; VIETTA, E. R Seguimen^ $\wedge$ to de enfermagem após alta hospitalar. BoL Sanit. Panan., 101(ó):638-47, 1986.

ROCHA, J. et ai. Tempo de pemmanência e cri^térios de alta para desnutridos. J. Pediatr., 51(3): 183-ó, 1981.

SCHRAEDER, B. D. Developmental progress in very low birth weight infants during the first year of life. Nurs. Res., 35(4):23742, 1986.

SCHRAEDER, B. D. et ai. The value of early home assessment in indentifying risk in children who were very low birth weight. Pediatr. Nurs., 16(3):168-272, 1990.

SEGRE, C. A. M. et ai. Efeitos das modificações de critérios para a alta de recém-nascidos de baixo peso. Rev. Paul. Pediatr, 4(15):138-40, 1986.

SIQUEIRA, A. A. E de. $\mathrm{e}^{\wedge} \mathrm{t}$ ai. instrumentos para o acompanhamento e avaliação do desenvolvimen^to infan^til na a^tenção primária de saúde. Rev. Bras. Cresc. Des. Hum., II(2):59-99, 1992.

ZILIOTTO, M. C. O esta^tuto da criança e do adolescente e a poli^tica de atendimento. Rev. Bras. Cresc. Des. Hum., II(1):15-46, 1992.

WENNBERG, R. R; AHLFORS, C. E. A. Discharging the jaundiced infant from the hospital: risk for the infant or physician? Pediatries, 81(1):175, 1988.

recebido em: 19/08/93 aprovado em: 21/03/94 